\title{
Conservation of high-flux backbone in alternate optimal and near-optimal flux distributions of metabolic networks
}

\author{
Areejit Samal
}

Received: 7 April 2009/ Accepted: 12 May 2009/Published online: 30 May 2009

(C) The Author(s) 2009. This article is published with open access at Springerlink.com

\begin{abstract}
Constraint-based flux balance analysis (FBA) has proven successful in predicting the flux distribution of metabolic networks in diverse environmental conditions. FBA finds one of the alternate optimal solutions that maximizes the biomass production rate. Almaas et al. have shown that the flux distribution follows a power law, and it is possible to associate with most metabolites two reactions which maximally produce and consume a given metabolite, respectively. This observation led to the concept of highflux backbone (HFB) in metabolic networks. In previous work, the HFB has been computed using a particular optima obtained using FBA. In this paper, we investigate the conservation of HFB of a particular solution for a given medium across different alternate optima and near-optima in metabolic networks of $E$. coli and $S$. cerevisiae. Using flux variability analysis (FVA), we propose a method to determine reactions that are guaranteed to be in HFB regardless of alternate solutions. We find that the HFB of a particular optima is largely conserved across alternate optima in E. coli, while it is only moderately conserved in $S$. cerevisiae. However, the HFB of a particular nearoptima shows a large variation across alternate near-optima in both organisms. We show that the conserved set of reactions in HFB across alternate near-optima has a large overlap with essential reactions and reactions which are both uniquely consuming (UC) and uniquely producing (UP). Our findings suggest that the structure of the metabolic network admits a high degree of redundancy and plasticity in near-optimal flow patterns enhancing system robustness for a given environmental condition.
\end{abstract}

\footnotetext{
A. Samal ( $\square)$

Max Planck Institute for Mathematics in the Sciences, Inselstr. 22, 04103 Leipzig, Germany

e-mail: samal@mis.mpg.de
}

Keywords Complex network - Flux balance analysis (FBA) - Alternate optima and near-optima · Flux variability analysis (FVA) · Flux plasticity

\section{Introduction}

The study of the structure and dynamics of complex biological networks is important for understanding the systemlevel behaviour of living organisms (Hartwell et al. 1999; Barabasi and Oltvai 2004). The metabolic network is one such physicochemical system inside the cell which can be viewed as a complex network consisting of metabolites involved in enzyme catalyzed reactions. Recent advances in the development of high-throughput data collection techniques coupled with systematic analysis of fully sequenced genomes has led to the reconstruction of organism-specific metabolic networks (Feist et al. 2009). Although the list of metabolic reactions along with the involved stoichiometries is largely known for many organisms, we currently lack the knowledge of most kinetic rate constants and enzyme concentrations in these networks. In the absence of detailed knowledge of various kinetic parameters, constraint-based modelling techniques like Flux balance analysis (FBA), which utilizes primarily structural information of the network in the form of stoichiometry of various reactions, have proven useful in predicting the quantitative behaviour of the cellular metabolism under different environmental conditions (Varma and Palsson 1994; Edwards et al. 2001; Kauffman et al. 2003; Price et al. 2004).

FBA is a computational technique which can be used to obtain a particular flux distribution for a given environmental condition that maximizes certain objective function which is usually taken to be the biomass production rate 
(see "Methods" section for details). However, for large scale metabolic networks, it has been shown that there are a large number of different flux distributions or alternate solutions for a given environmental condition with the exact same value for the objective function (Lee et al. 2000; Mahadevan and Schilling 2003; Reed and Palsson 2004). Thus, FBA finds one of many possible alternate optimal solutions for a given environmental condition. Mixed integer linear programming (MILP) has been used to enumerate different alternate optimal flux distributions for a given environmental condition (Lee et al. 2000; Reed and Palsson 2004). However, for large scale metabolic networks, the number of alternate optimal solutions has been shown to be extremely large, and it is computationally infeasible using MILP to enumerate all possible alternate optima (Reed and Palsson 2004). Although, it is not feasible to enumerate all alternate optima, flux variability analysis (FVA) (Mahadevan and Schilling 2003) can be used to determine the minimum and maximum flux value of each reaction across the set of alternate optimal solutions for a given environmental condition. Specifically, using FVA, we can determine reactions that can have a nonzero flux in some alternate optima and the reactions whose flux vary across different alternate optima for a given environmental condition.

In the FBA approach, we obtain a particular solution by imposing the optimality criterion wherein a flux distribution satisfying the governing constraints along with maximal biomass production rate is predicted as the phenotype for a given environmental condition. However, the optimal flux distribution with maximal biomass production may not always explain the observed behaviour for a given environmental condition (Ibarra et al. 2002; Segre et al. 2002; Fong et al. 2005; Schuetz and Kuepfer 2007; Schuster et al. 2008). Further, wet lab experiments have shown that the organism may initially grow suboptimally under certain media, i.e., the observed growth rate is less than the maximum value predicted by FBA, and then evolve to grow at the predicted optimal rate after several generations (Ibarra et al. 2002; Fong et al. 2005). It is plausible that there are multiple objectives that the cell is trying to optimize to different extent under different environments. Thus, the metabolic network may operate under suboptimal growth conditions under certain environmental conditions. Hence, it is important to study the suboptimal flux distributions, especially, near-optimal flux distributions in metabolic networks for different environmental conditions.

Using the optimal solution predicted by FBA, Almaas et al. (2004) have shown that the distribution of reaction fluxes for a given environmental condition follows a power law in the E. coli metabolic network. The observed power law distribution implies an inhomogeneity in the reaction network usage with most reactions having small fluxes and a few reactions having high fluxes. Almaas et al. (2004) showed that the heterogeneity of overall flux distribution is also valid to a large extent at the level of individual metabolites. Further, for most metabolites, they were able to identity two reactions that dominate the production and consumption of a metabolite, respectively. Using this property at the level of an individual metabolite, Almaas et al. (2004) have proposed a simple algorithm to construct a subnetwork referred to as the "high-flux backbone" (HFB) which contains the locally maximal flow paths for a given environmental condition in the network. The HFB for a given environmental condition was shown to form a giant connected cluster with large overlap with classical pathways described in biochemical literature. It has also been suggested that the HFB can be used as a network reduction algorithm.

In this paper, we have studied the activity and flux variability of different reactions across alternate flux distributions for a given medium under optimal and suboptimal growth conditions in metabolic networks of $E$. coli and S. cerevisiae. Almaas et al. (2004) have used a single solution obtained using FBA from the set of different alternate optima to compute the HFB of the metabolic network for a given medium. In this paper, we have investigated in detail the effect of alternate optima on the set of reactions in HFB computed using a single solution for a given medium in E. coli and S. cerevisiae. We have further investigated the effect of alternate suboptimal solutions corresponding to near-optimal growth condition on the set of reactions in HFB computed using a single suboptimal solution for a given medium in the two organisms.

\section{Methods}

Flux balance analysis

Flux balance analysis is a computational modelling technique which can be used to obtain a prediction for the fluxes of all reactions in the metabolic network and growth rate of the organism (Varma and Palsson 1994; Kauffman et al. 2003; Price et al. 2004). The main assumptions made in the technique of FBA are: (a) the network reaches a steady state for any given environmental condition wherein the concentration of all internal metabolites and velocities of all reactions are constant, and (b) the cell tries to adjust its intracellular machinery or reaction fluxes so as to maximize its growth rate or biomass production. The key requirement for implementing FBA is the information about the stoichiometry of all metabolic reactions known to occur in an organism. The complete list of reactions along with their stoichiometry is encapsulated in the 
stoichiometric matrix $\mathbf{S}$ of dimensions $m \times n$ where $m$ denotes the number of metabolites and $n$ denotes the number of reactions. In any metabolic steady state, each metabolite achieves a dynamic mass balance and the vector $\mathbf{v}=\left(v_{1}, v_{2}, \ldots, v_{n}\right)^{T}$ representing the fluxes through each reaction must satisfy the equation

$\mathbf{S} \cdot \mathbf{v}=0$

so as to not violate mass conservation. Equation 1 gives us a system of linear equations relating various fluxes in the network. In addition to linear stoichiometric constraints, there exist thermodynamic constraints that may render certain reactions irreversible and flux capacity constraints which impose limitations on maximum flux through certain reactions. These additional constraints can be used to further limit the space of allowable fluxes given by Eq. 1. To obtain a particular solution from the space of allowable fluxes, linear programming (LP) is used to find a flux distribution in the allowable space that gives the optimal value for an objective function. The most commonly used objective function is the maximization of biomass production rate. The LP formulation of the FBA approach is as follows:

$$
\begin{aligned}
\text { Maximize } Z & =\mathbf{c}^{\mathbf{T}} \mathbf{v} \\
\text { such that } \mathbf{S} \cdot \mathbf{v} & =0 \\
\alpha & \leq v_{i} \leq \beta
\end{aligned}
$$

where vector $\mathbf{c}$ corresponds to the objective function, $\alpha$ and $\beta$ are the lower and upper limits of individual flux values.

\section{Mixed integer linear programming}

For most large scale metabolic networks, FBA finds one of many possible alternate optimal flux distributions that have the same objective value for a given environmental condition (Lee et al. 2000; Mahadevan and Schilling 2003; Reed and Palsson 2004). We can use MILP to enumerate different alternate optima. We have used here the recursive MILP algorithm proposed by Reed and Palsson (2004) to enumerate different alternate optima. In this method, in addition to constraints in the LP formulation of FBA given by Eq. 2, the following new constraints are imposed to obtain different solutions:

$$
\begin{aligned}
\sum_{i \in N Z^{J-1}} y_{i} & \geq 1 \\
\sum_{i \in N Z^{J}} w_{i} & \geq\left|N Z^{k}\right|-1, \quad k=1 \ldots J-1 \\
y_{i}+w_{i} & \leq 1, \quad \forall i \\
\alpha \cdot w_{i} & \leq v_{j} \leq \beta \cdot w_{i} \quad \forall i
\end{aligned}
$$

In this method each reaction $i$ in the network is associated with two binary variables $y_{i}$ and $w_{i} . \quad N Z^{J}$ represents the set of reactions with nonzero flux value in the solution at iteration $J$. Given the constraints in Eq. 3, if the binary variable $y_{i}=1$ then $w_{i}=0$, which forces both upper and lower bounds for reaction $i$ to be zero. Thus, setting $y_{i}=1$ forces the reaction $i$ to be removed from the basis of the next iteration. In this recursive algorithm, at each iteration $J$, at least one of the nonzero fluxes of the previous solution $\left(N Z^{J-1}\right)$ is removed from the basis of the current iteration. The MILP problem given by Eqs. 2 and 3 is then solved again to generate a different alternate solution.

Flux variability analysis

The number of alternate optima in large scale metabolic networks is extremely large, and it can be computationally infeasible to enumerate all alternate optima. FVA (Mahadevan and Schilling 2003) is an alternative technique which can be used to determine the flux variability associated with each reaction in the complete set of alternate optima for a given medium. Initially, the maximum value of the objective function, i.e., $Z_{o b j}$, is determined. This is followed by solving two LP problems for each reaction $i$ in the network. The LP formulation of the FVA approach is as follows:

Case 1:

$$
\begin{aligned}
& \text { Maximize } v_{i} \\
& \text { such that } \mathbf{S} \cdot \mathbf{v}=0 \\
& \qquad \mathbf{c}^{\mathbf{T}} \mathbf{v}=Z_{o b j} \\
& \alpha \leq v_{i} \leq \beta \quad \text { for } i=1 \ldots n
\end{aligned}
$$

Case 2:

$$
\begin{aligned}
& \text { Minimize } v_{i} \\
& \text { such that } \mathbf{S} \cdot \mathbf{v}=0 \\
& \mathbf{c}^{\mathbf{T}} \mathbf{v}=Z_{o b j} \\
& \alpha \leq v_{i} \leq \beta \text { for } i=1 \ldots n .
\end{aligned}
$$

In the first case, the maximum flux through each reaction that supports optimal growth rate $Z_{o b j}$ is determined. In the second case, the minimum flux through each reaction that supports optimal growth rate $Z_{o b j}$ is determined. The difference of maximum and minimum value that supports optimal growth rate for each reaction gives the flux variability of that reaction. A slight modification of the LP problem given by Eqs. 4 and 5 is able to generate the flux variability under suboptimal conditions. The suboptimal condition corresponding to near-optimality studied here requires the following change in Eqs. 4 and 5: Replace the condition $\mathbf{c}^{\mathbf{T}} \mathbf{v}=Z_{o b j}$ with $\mathbf{c}^{\mathbf{T}} \mathbf{v} \geq 0.99 Z_{o b j}$.

\section{Reactions involved in futile cycles}

We used a recently published algorithm by Wright and Wagner (2008) to compute the reactions in E. coli and 
$S$. cerevisiae metabolic network that are involved in internal cycles (Beard et al. 2002; Price et al. 2002) of size $\geq 3$. The number of reactions involved in internal cycles of size $\geq 3$ in E. coli and $S$. cerevisiae was obtained to be 22 and 60, respectively. Using METATOOL version 5.0 (von Kamp and Schuster 2006), we obtained a total of 9 and 28 internal cycles of size $\geq 3$ in the metabolic network of E. coli and S. cerevisiae, respectively. In order to avoid these internal cycles, we followed the standard procedure of constraining at least one reaction flux in each internal cycle to zero. By examining the different internal cycles of size $\geq 3$, we decided to constrain the flux of following reactions: ABUTt2, ACCOAL, ADK1, GLUT4, PROt4, SERt4, THRt4 and VPAMT in the E. coli metabolic network iJR904 (Reed et al. 2003) and the flux of following reactions: ASPTAm, ACONTm, DCMPDA, FRDcm, GHMT2r, GLUT5m, MALt2r, NDPK1, NDPK8, NDPK9, PHCDm, SHSL1, SUCCtm, SUCCFUMtm, SUCD2_u6m and UGLT in the $S$. cerevisiae metabolic network iND750 (Duarte et al. 2004) to zero to avoid futile cycles. The abbreviations of the reactions in the two metabolic networks mentioned above are same as in the databases iJR904 and iND750, respectively.

\section{High-flux backbone}

The method to determine HFB for a given medium using a particular solution is as follows (Almaas et al. 2004):

(a) For each metabolite in the network, remove all reactions except the reaction that produces the metabolite with largest flux and the reaction that consumes the metabolite with largest flux. The metabolites that are either not produced (consumed) are not taken into account.

(b) If metabolite $A$ is an educt and metabolite $B$ is a product of reaction $R$, and further $R$ maximally consumes $A$ and maximally produces $B$, then reaction $R$ is part of the HFB.

Since, the set of reactions in HFB of a particular solution may change if it was obtained from a different alternate solution, we here propose a simple method using FVA to determine the set of reactions that are guaranteed to be in HFB of all alternate solutions for a given medium. Our method is as follows:

(a) Using FVA, we obtain the minimum and maximum flux of each reaction $R$ in the network that can support optimal growth for a given minimal medium.

(b) We designate reaction $R$ as producing (consuming) metabolite $A$ with largest flux across all alternate optima, if the minimum flux for $R$ obtained using
FVA is greater than the maximum flux for each reaction other than $R$ that produces (consumes) $A$.

(c) For each metabolite in the network, remove all reactions except the reaction that produces the metabolite with largest flux and the reaction that consumes the metabolite with largest flux across all alternate optima.

(d) If metabolite $A$ is an educt and metabolite $B$ is a product of reaction $R$, and further $R$ maximally consumes $A$ and maximally produces $B$ across all alternate optima, then reaction $R$ is contained in HFB of all alternate optima.

Our method described here for alternate optimal solutions can also be used to determine the set of reactions that are guaranteed to be in HFB of all alternate near-optimal solutions.

\section{Results and discussion}

In this section, we present results from our study of the two metabolic networks: E. coli [version iJR904 (Reed et al. 2003)] and S. cerevisiae [version iND750 (Duarte et al. 2004)]. The two networks can be downloaded from the BiGG database: http://bigg.ucsd.edu/. The E. coli metabolic network iJR904 contains 761 metabolites and 931 reactions and the $S$. cerevisiae metabolic network iND750 contains 1,061 metabolites and 1,149 reactions. For each organism, the two databases additionally provide a biomass production reaction which gives the relative contribution of key metabolites towards cellular biomass. We have investigated here the two metabolic networks under different environmental conditions corresponding to 89 and 43 aerobic minimal media for E. coli and $S$. cerevisiae, respectively. Using FBA, it has been predicted elsewhere that this set of 89 and 43 aerobic minimal media for $E$. coli and $S$. cerevisiae, respectively, considered here are the only aerobic minimal media that can support nonzero growth in the two networks (Samal et al. 2006).

Activity and flux variability of reactions under optimal and near-optimal conditions

We can determine the maximum and minimum flux through each reaction that supports optimal growth in a minimal medium using FVA (see "Methods" section for details). A reaction is considered to be possibly active in a medium across alternate optima, if the maximum flux value that supports optimal growth is nonzero. A reaction is considered to have a variable flux in a medium across alternate optima, if the difference between maximum and 
minimum flux value that supports optimal growth is nonzero.

Using FVA, we determined the number of reactions that can be possibly active and the reactions with variable flux across alternate optima for each of the 89 different minimal media in the E. coli metabolic network. The number of reactions that can be possibly active in a medium varies between 278 and 332 reactions across the 89 minimal media. The number of reactions with variable flux in a medium varies between 34 and 83 reactions across the 89 minimal media. These results for the E. coli metabolic network are shown in Fig. 1a. We find that $11-25 \%$ of the reactions that can be possibly active in a given medium to have variable flux across alternate optima for that medium. The results obtained here for the E. coli metabolic network using FVA for 89 different aerobic minimal media are consistent with the results obtained earlier by Reed and Palsson (2004) using a larger set of alternate optima for 136 different aerobic and anaerobic minimal media.

We also determined the number of reactions that can be possibly active and the reactions with variable flux across alternate optima for each of the 43 different minimal media in the $S$. cerevisiae metabolic network. These results for the S. cerevisiae metabolic network are shown in Fig. 2a. We find that $22-69 \%$ of the reactions that can be possibly active in a given medium to have variable flux across alternate optima for that medium in S. cerevisiae. For one of the 43 minimal media, i.e., arginine aerobic minimal medium, we find a much larger number of possibly active reactions and reactions with variable flux across alternate optima compared to other minimal media (cf. Fig. 2a). A closer look at the data for arginine minimal medium showed that many transport reactions involved in excretion of ammonia, guanine, urea and xanthine have a variable flux across alternate optima. This observation explains the larger flexibility under arginine minimal medium within the $S$. cerevisiae metabolic network to achieve optimal growth rate compared to other media. Even if we exclude the results for the arginine minimal medium, we find that the flux variability for a typical minimal medium in $S$. cerevisiae is greater than the flux variability for a typical minimal medium in $E$. coli under optimal growth
Fig. 1 Activity and flux variability of reactions under a optimal growth, b near-optimal growth ( $\geq 99 \%$ of the optimal rate) and $\mathbf{c}$ suboptimal growth ( $\geq 5 \%$ of the optimal rate) for 89 different minimal media in the E. coli metabolic network. Each bar corresponds to one of the 89 different minimal media. The height of the bar gives the number of reactions that can be possibly active and the height upto which the bar is filled with grey colour gives the number of reactions whose flux vary across alternate solutions for that medium

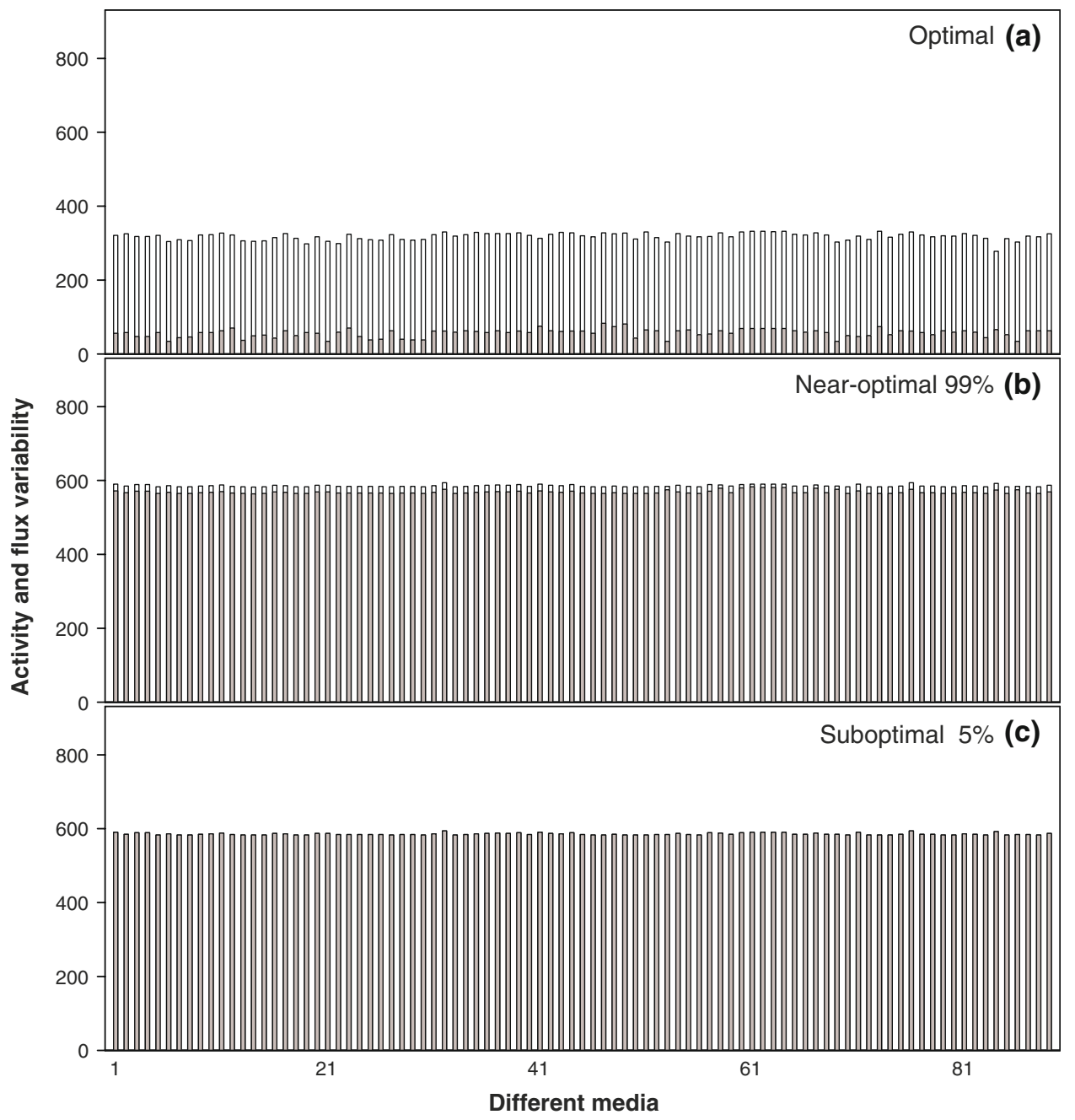


Fig. 2 Activity and flux variability of reactions under a optimal growth, b nearoptimal growth ( $\geq 99 \%$ of the optimal rate) and c suboptimal growth $(\geq 5 \%$ of the optimal rate) for 43 different minimal media in the $S$. cerevisiae metabolic network. Each bar corresponds to one of the 43 different minimal media. The height of the bar gives the number of reactions that can be possibly active and the height upto which the bar is filled with grey colour gives the number of reactions whose flux vary across alternate solutions for that medium

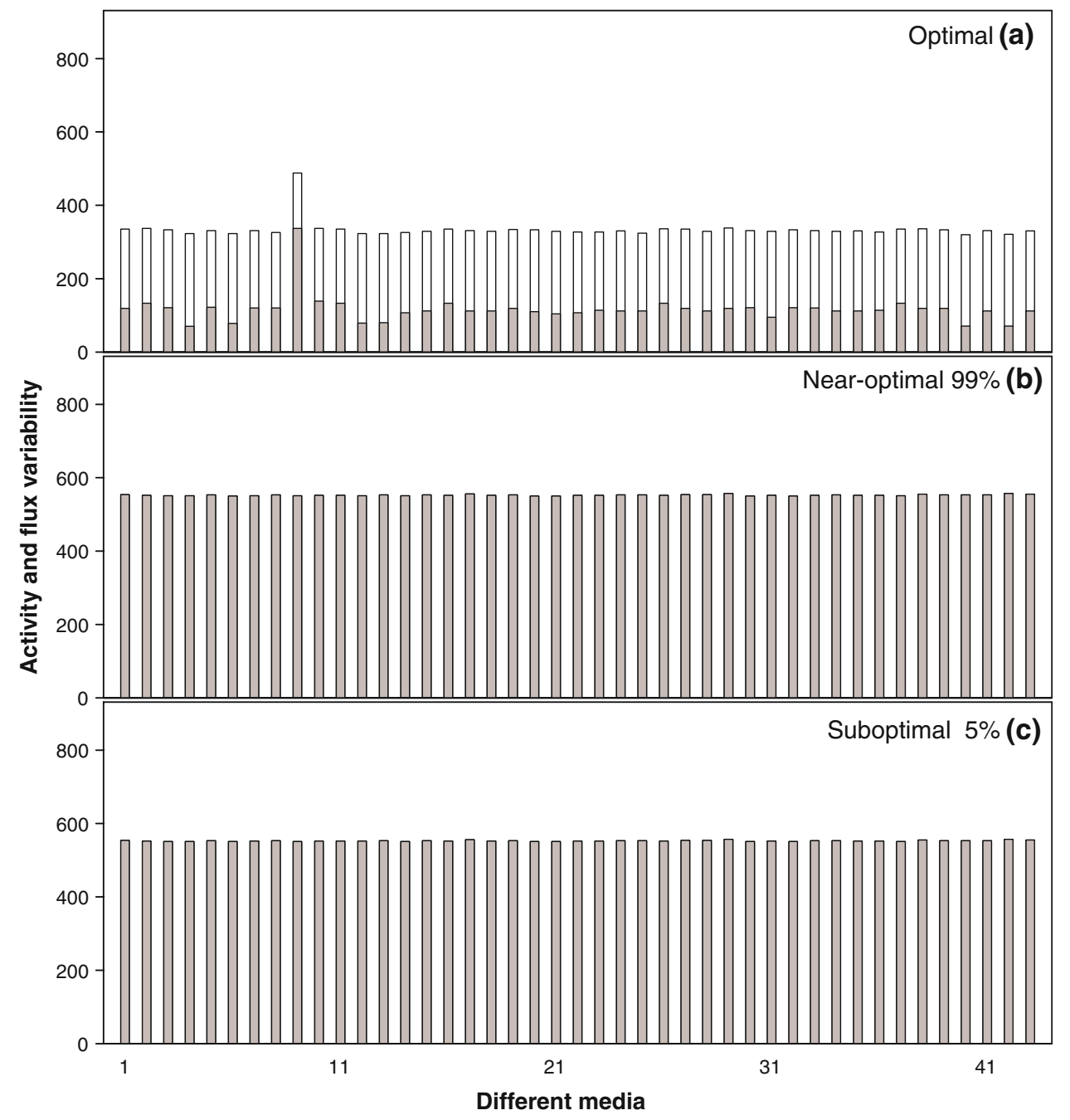

condition. This finding suggests a more evolved structure of the metabolic network inside the eukaryote $S$. cerevisiae with greater redundancy compared to that of the prokaryote E. coli.

We investigated both metabolic networks under two suboptimal scenarios: (a) where the growth rate is $\geq 99 \%$ of the optimal growth rate and (b) where the growth rate is $\geq 5 \%$ of the optimal growth rate. The first of the two suboptimal scenarios will be referred to as 'near-optimal condition' as we account for all flux distributions that allow growth at $\geq 99 \%$ of the optimal rate. In the second suboptimal scenario, we account for all flux distributions which allow growth at $\geq 5 \%$ of the optimal rate. We used FVA to determine the number of possibly active reactions and the reactions with variable flux in a medium across alternate suboptimal solutions for different minimal media. From here onwards, we refer to the set of alternate optimal flux distributions for a given minimal medium in short as 'alternate optima'. For the first suboptimal scenario, we refer to the set of alternate suboptimal flux distributions which have near-optimal growth rate for a given minimal medium in short as 'alternate near-optima'. For the second suboptimal scenario, we refer to the set of alternate suboptimal flux distributions which have growth rate $\geq 5 \%$ of the optimal rate for a given minimal medium in short as 'alternate suboptima'.

Using FVA, we determined the number of reactions that can be possibly active and the reactions with variable flux in the two suboptimal scenarios for each of the 89 different minimal media in the E. coli metabolic network. The number of reactions that can be possibly active in a given medium is obtained to be close to 600 in both suboptimal scenarios. Almost all reactions which can be possibly active have variable flux across alternate near-optima in the first suboptimal scenario. Further, all reactions which can be possibly active have variable flux across alternate suboptima in the second suboptimal scenario. These results for the E. coli metabolic network are shown in Fig. 1b and c. We find that most reactions which can be possibly active in a given medium can have a variable flux across alternate solutions in near-optimal growth condition (cf. Fig. 1b). We found similar results for both suboptimal conditions in 
the $S$. cerevisiae metabolic network (cf. Fig. 2b, c). We emphasize that although the set of reactions with variable flux under both suboptimal scenarios is almost the same, the amount of flux variability of a reaction is larger in the second suboptimal scenario compared to the first nearoptimal scenario.

By comparing the number of possibly active reactions in each minimal medium investigated in E. coli (cf. Fig. 1a, b) and S. cerevisiae (cf. Fig. 2a, b) under optimal and nearoptimal conditions, we can clearly see that the number is significantly more in the near-optimal condition. Thus, a larger set of reactions can be recruited under near-optimal condition compared to strictly optimal condition. This suggests a high degree of flux plasticity in the metabolic network under near-optimal condition. In a recent paper, Nishikawa et al. (2008) have shown that the maximization of a linear objective function, such as growth rate or ATP production, in FBA to obtain a particular flux distribution leads to significantly lower number of active reactions under optimal condition compared to typical non-optimal states. They attribute this massive spontaneous reaction silencing to the irreversibility of reactions and cascade of inactivity propagated by irreversible reactions in the network. Our results obtained using FVA add to the results obtained by Nishikawa et al. (2008). We have shown here that, even in near-optimal growth conditions, the number of possibly active reactions and the reactions with variable flux across alternate near-optima is much larger compared to strictly optimal scenario.

We end this subsection by mentioning an important methodological detail. While implementing FVA, we need to account for reactions that are involved in futile internal cycles. An internal cycle or Type III extreme pathway is a set of internal reactions that can satisfy the steady state mass balance condition without involvement of any exchange flux (Beard et al. 2002; Price et al. 2002). Such cycles are thermodynamically infeasible and need to be eliminated in any steady state analysis. We determined all internal cycles in the two metabolic networks studied here, and based on that, decided to constrain the flux of 8 reactions in E. coli and 16 reactions in S. cerevisiae to zero to avoid futile cycles in our simulations (see "Methods" section for details).

High-flux backbone under optimal and near-optimal conditions

In the previous subsection, we have shown that there is variation in the activity as well as flux value of reactions across the alternate optima for a given medium in both E. coli and S. cerevisiae. Further, we have shown that the number of possibly active reactions and reactions with variable flux across alternate near-optima is significantly higher in near-optimal condition compared to the strictly optimal case. In this subsection, we investigate the effect of alternate flux distributions on the set of reactions in the HFB obtained using a particular solution in both optimal and near-optimal conditions.

Using MILP (Lee et al. 2000; Reed and Palsson 2004), we obtained 100 alternate optima and 100 alternate nearoptima for each of the 89 aerobic minimal media in E. coli and 43 aerobic minimal media in $S$. cerevisiae (see "Methods" section for details). For each minimal medium investigated, we obtained the 100 HFB corresponding to 100 alternate optima. We then calculated the average number of reactions in HFB across the alternate optima for each medium. These results for the 89 minimal media in $E$. coli and 43 minimal media in $S$. cerevisiae under optimal growth conditions are shown in Figs. $3 \mathrm{a}$ and $4 \mathrm{a}$, respectively. Similarly, we obtained the average number of reactions in HFB across the alternate near-optima for each medium. These results are shown for E. coli and S. cerevisiae in Figs. $3 \mathrm{~b}$ and $4 \mathrm{~b}$, respectively.

In case of both $E$. coli and $S$. cerevisiae, for a given medium, we found the set of reactions in HFB corresponding to different alternate optima to differ from each other. This was also true for the set of reactions in the HFB corresponding to different alternate near-optima. However, the number of alternate optima or alternate near-optima sampled here is too small, and the data cannot be used to determine the overlap among the reactions in HFB corresponding to different alternate solutions for a given medium. Thus, we devise a simple method using FVA to determine the set of reactions which are guaranteed to be in any HFB corresponding to different alternate solutions for a given medium (see "Methods" section for details). We used this method to determine the set of reactions that are guaranteed to be in HFB corresponding to any alternate optima for a given medium in the two networks. We also obtained the set of reactions that are guaranteed to be in HFB corresponding to different alternate near-optima for a given medium in the two networks. These results are shown for the 89 minimal media in E. coli in Fig. $3 \mathrm{a}$ and b and for the 43 minimal media in $S$. cerevisiae in Fig. 4a and $b$.

We find that at least $3-13 \%$ of reactions in HFB corresponding to a particular optima for a given medium are not guaranteed to be in HFB corresponding to some alternate optima for that medium in the E. coli metabolic network (cf. Fig. 3a). However, at least $34-42 \%$ of reactions in HFB corresponding to a particular near-optima for a given medium are not guaranteed to be in HFB corresponding to some alternate near-optima for that medium in E. coli (cf. Fig. 3b). Similarly, we find that at least 11-45\% of reactions in HFB corresponding to a particular optima for a given medium are not guaranteed to be in HFB 
Fig. 3 Histogram of the average number of reactions in HFB under a optimal growth and $\mathbf{b}$ near-optimal growth for 89 different minimal media in the $E$. coli metabolic network. Each bar corresponds to one of the 89 different minimal media. The height of the bar gives the average number of reactions in the HFB computed using 100 alternate solutions obtained using MILP for each minimal medium. The height upto which the bar is filled with grey colour gives the number of reactions which are guaranteed to be in HFB of all alternate solutions for that medium

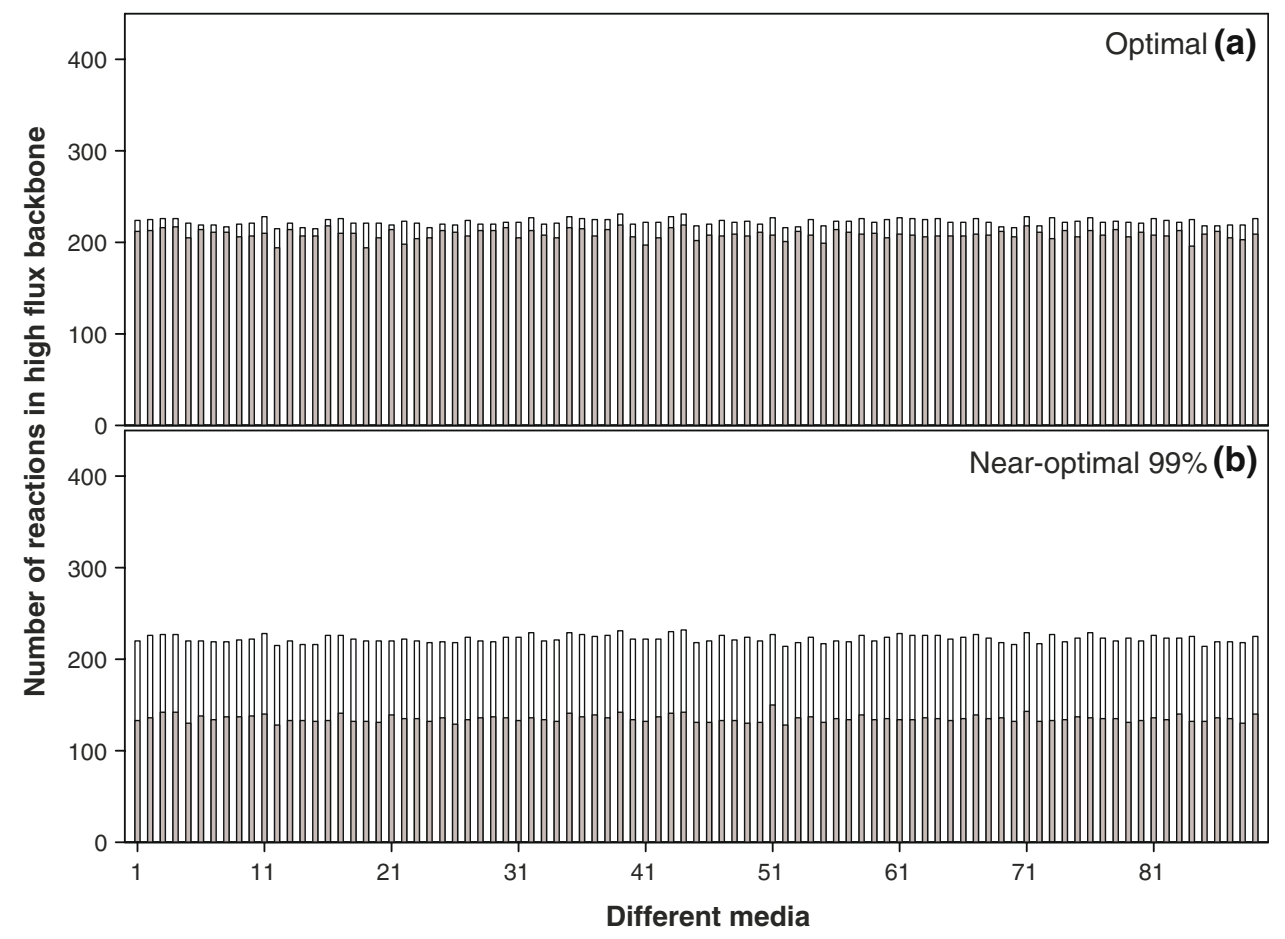

Fig. 4 Histogram of the average number of reactions in HFB under a optimal growth and $\mathbf{b}$ near-optimal growth for 43 different minimal media in the $S$. cerevisiae metabolic network. Each bar corresponds to one of the 43 different minimal media. The height of the bar gives the average number of reactions in the HFB computed using 100 alternate solutions obtained using MILP for each minimal medium. The height upto which the bar is filled with grey colour gives the number of reactions which are guaranteed to be in HFB of all alternate solutions for that medium

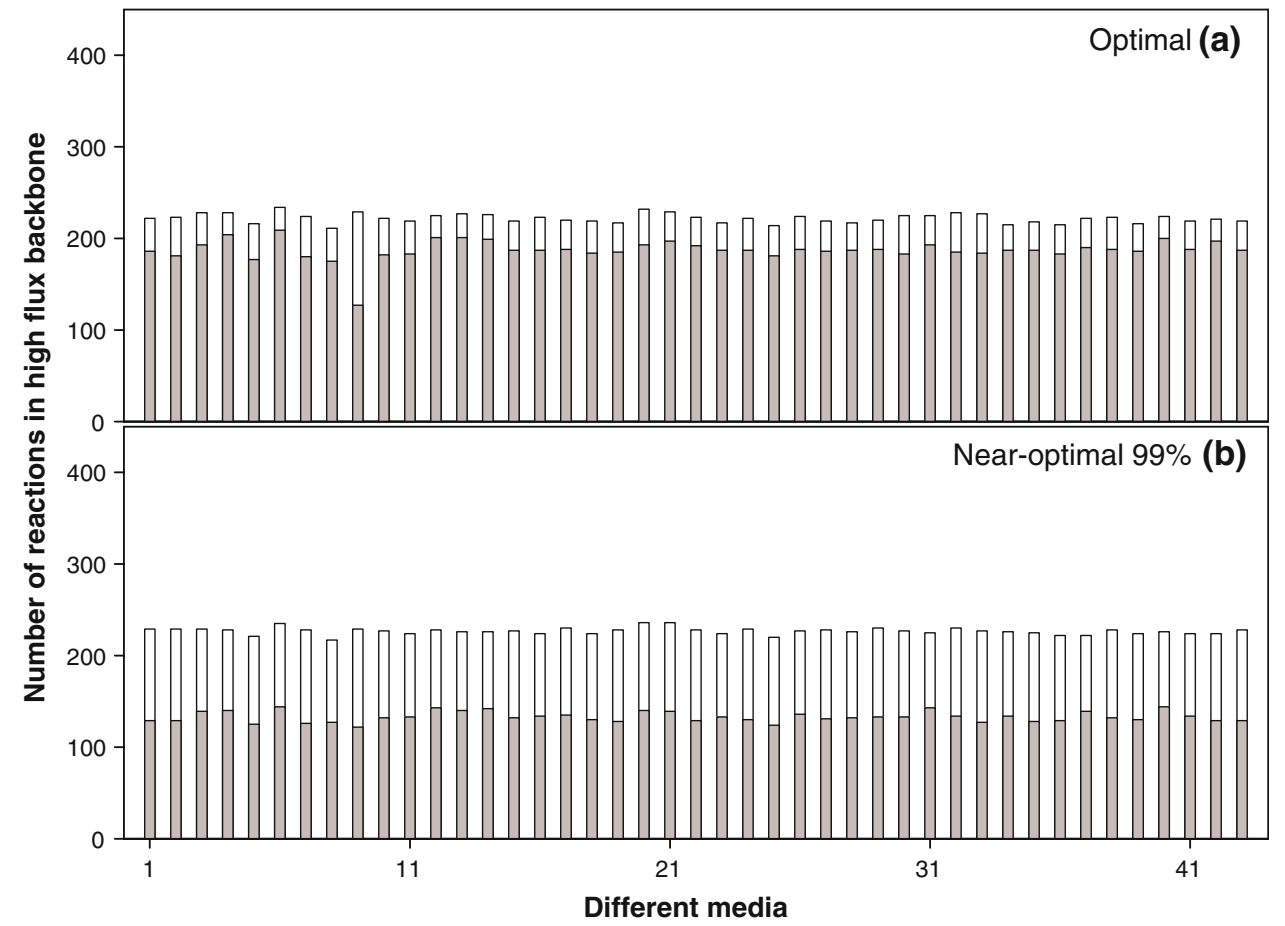

corresponding to some alternate optima for that medium in the $S$. cerevisiae metabolic network (cf. Fig. 4a). Even if we discount the arginine minimal medium, which has an uncharacteristically larger flux variability in $S$. cerevisiae under optimal conditions (cf. Fig. 2a), still at least 11-20\% of reactions in HFB corresponding to a particular optima for a given medium are not guaranteed to be in HFB corresponding to some alternate optima for that medium. Further, at least $36-47 \%$ of reactions in HFB corresponding to a particular near-optima are not guaranteed to be in HFB corresponding to some alternate near-optima for that medium in S. cerevisiae (cf. Fig. 4b).

Note that we have used the simplex method to solve the LP problem in order to sample different alternate optima 
and near-optima. Simplex method tries to return a particular solution or flux distribution from the set of alternate solutions that has least number of nonzero reaction fluxes in it (Almaas 2007). Thus, our results obtained using MILP regarding the average number of reactions in HFB corresponding to a particular solution computed using 100 alternate solutions for a given medium may change with exhaustive sampling. However, our results obtained using FVA regarding the set of reactions that are guaranteed to be in HFB corresponding to any alternate optima or suboptima for a given medium is independent of sampling.

We have shown here that the set of reactions in HFB depends upon the particular solution chosen from the set of alternate optima to compute it in both $E$. coli and $S$. cerevisiae. Under optimal condition, only $3-11 \%$ of the reactions in HFB of a particular solution are not guaranteed to be in HFB of some other alternate optima in E. coli which is relatively small. But the corresponding fraction in $S$. cerevisiae for optimal condition is much higher. Also, we have shown for near-optimal condition, greater than $34 \%$ of the reactions in HFB of a particular near-optima are not guaranteed to be in HFB of some other alternate nearoptima in both organisms. Thus, the set of reactions in HFB significantly varies under near-optimal conditions across alternate near-optima for a given medium in both organisms. These findings suggest that the flow patterns in metabolic networks for a given medium in near-optimal conditions is much more complex and degenerate compared to that under strictly optimal conditions.

Our findings also imply that even for a given medium the structure of the metabolic network has evolved so as to allow both structural and flux plasticity (Almaas et al. $2005)$ in the level of reaction usage to achieve the same optimal or near-optimal growth objective through multiple flux distributions. Structural plasticity associated with a given medium can result in alternate equally efficient pathways or metabolic routes to be recruited in different alternate solutions. Flux plasticity associated with a given medium can result in different value of reaction fluxes in different alternate solutions. Both structural and flux plasticity associated with alternate flux distributions for a given medium renders the set of reactions in HFB of a particular solution for that medium variable across alternate solutions. However, we still find that, under near-optimal condition, at least $53 \%$ of the reactions in HFB of a particular solution are conserved across HFB of any alternate near-optima for a given medium in both organisms. In contrast, we have shown in the last subsection for nearoptimal condition that, almost all reactions that can be possibly active for a given minimal medium have a variable flux across alternate near-optima for that medium.

We now shed light on why at least $53 \%$ of the reactions in HFB of a particular solution are conserved across HFB of any alternate near-optima for a given medium when almost all reactions that can be possibly active for that medium show variation in their flux value. Since, the set of conserved reactions across HFB of any alternate nearoptima are always active for a given medium, it is natural to expect this set of reactions to have a high overlap with essential reactions for growth in that medium. We find that on average $90 \%$ of reactions guaranteed to be in HFB of alternate near-optima are essential for a given medium in $E$. coli while the corresponding fraction in $S$. cerevisiae is $80 \%$ (cf. Fig. 5). Thus, the conserved part of HFB across alternate solutions for a given medium has high density of essential reactions.

It has been shown elsewhere that almost all essential reactions in the metabolic network get explained by their association with low degree uniquely produced (UP) or uniquely consumed (UC) metabolite (Samal et al. 2006; Palumbo et al. 2005). Notice that if a given reaction consumes a UC metabolite and produces a UP metabolite, and the reaction has a nonzero flux value across all alternate near-optima for a given medium, then it is guaranteed to be in HFB of any alternate near-optima. We find that on average $80 \%$ of reactions guaranteed to be in HFB of alternate nearoptima consume a UC metabolite and produce a UP metabolite for a given medium in $E$. coli while the corresponding fraction for $S$. cerevisiae is $77 \%$ (cf. Fig. 6). Thus, the conserved part of HFB across alternate solutions for a given medium has high density of reactions that satisfy both UP and UC property. In determining UP and UC metabolites for a given medium, we removed the blocked reactions (Schuster and Schuster 1991; Burgard et al. 2004) for that medium as outlined in Samal et al. (2006).

The above mentioned results show that most reactions that are conserved in HFB regardless of alternate nearoptima for a given medium get explained by the absence of multiple paths to carry out certain processes in the metabolic network. Our findings also suggests that, in most cases, if a metabolite has multiple reactions producing it and multiple reactions consuming it, we cannot associate the metabolite with a single reaction that produces it maximally across all alternate near-optima and a single reaction that consumes it maximally across all alternate near-optima. These results show that, under near-optimal conditions, the metabolic network of E. coli and S. cerevisiae exhibit a high degree of flux plasticity for a given minimal medium contributing towards the robustness of the system.

\section{Conclusions}

In this paper, we have investigated the effect of alternate optima and near-optima for a given medium on the set of 
Fig. 5 Histogram of the fraction of reactions guaranteed to be in HFB of any alternate near-optima that are essential for a 89 minimal media in E. coli and b 43 minimal media in $S$. cerevisiae

Fig. 6 Histogram of the fraction of reactions guaranteed to be in HFB of any alternate near-optima that satisfy both UP and UC property for a 89 minimal media in E. coli and b 43 minimal media in S. cerevisiae
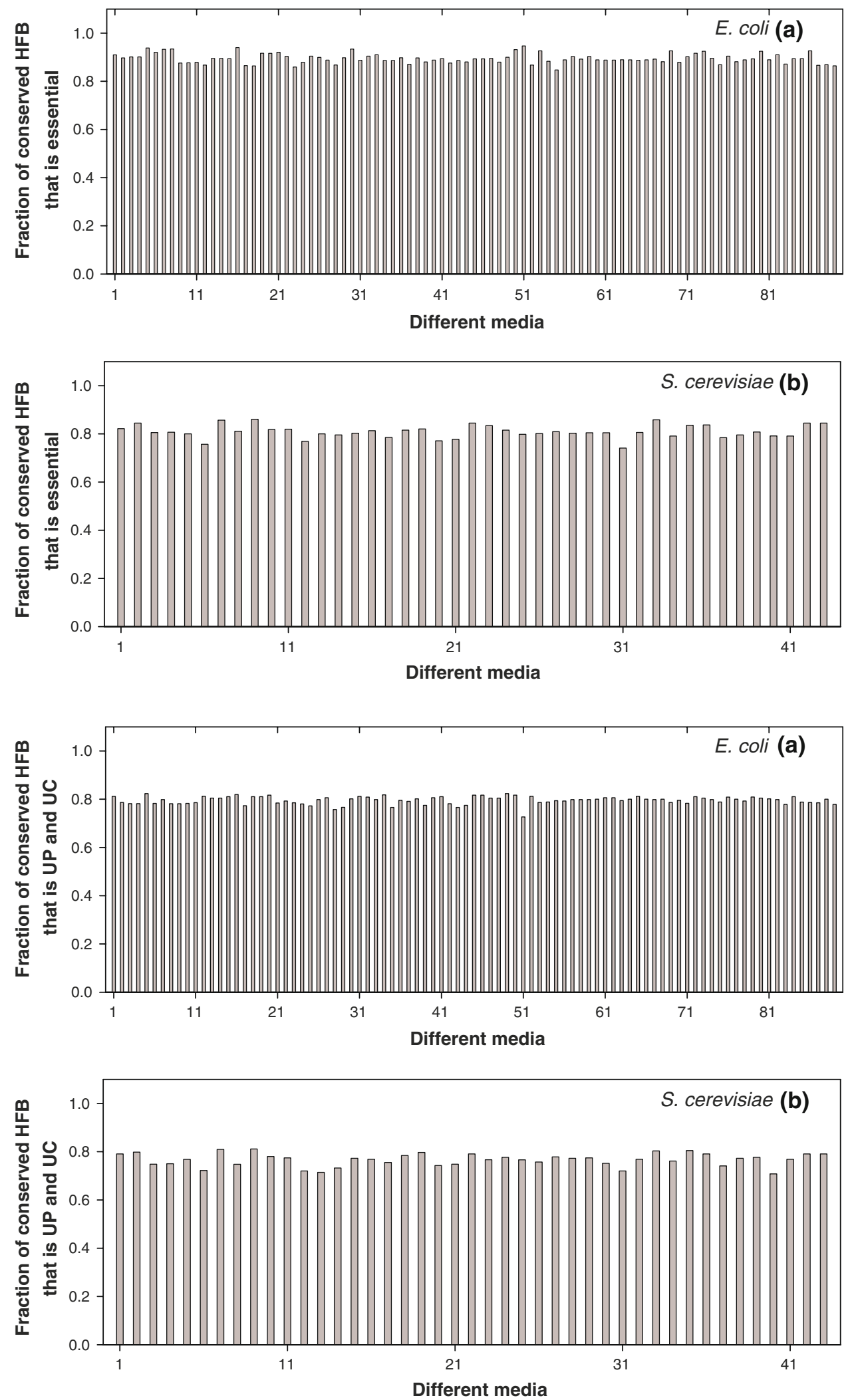

reactions in the HFB computed using a particular solution for that medium in the E. coli and S. cerevisiae metabolic network. Using FVA, we have shown here that the number of reactions that can be possibly active and reactions with variable flux across alternate solutions for a given medium under strictly optimal condition is significantly less than 
that in near-optimal condition for both metabolic networks. Since, the set of reactions in the HFB of a particular solution may vary across different alternate solutions for a given medium, we devised a simple method using FVA to determine the set of reactions which are guaranteed to be in HFB of any alternate solution for a given medium. We find that the set of reactions in HFB of particular optima for a given medium shows small variation across alternate optima for that medium in E. coli and moderate variation in $S$. cerevisiae. However, this variation is significantly large in both organisms when we consider the alternate nearoptima for a given medium. We show that the majority of reactions that are guaranteed to be in HFB of any alternate near-optima for a given medium are also essential for growth in that medium. We also show that majority of reactions in the conserved HFB set across alternate nearoptima for a given medium also satisfy both the uniquely consuming (UC) and uniquely producing (UP) property. Our results show that the structure of the metabolic network allows a high degree of structural and flux plasticity within a given environmental condition under near-optimal conditions which enhances the robustness of metabolic networks in both organisms.

Acknowledgements The author wishes to thank Andreas Wagner and Thimo Rohlf for discussions, and Pierre-Yves Bourguignon for his feedback on the manuscript. He gratefully acknowledges a postdoctoral fellowship from the Max Planck Institute for Mathematics in the Sciences, Leipzig.

Open Access This article is distributed under the terms of the Creative Commons Attribution Noncommercial License which permits any noncommercial use, distribution, and reproduction in any medium, provided the original author(s) and source are credited.

\section{References}

Almaas E (2007) Optimal flux patterns in cellular metabolic networks. Chaos 17:026107

Almaas E, Kovacs B, Vicsek T, Oltvai ZN, Barabasi AL (2004) Global organization of metabolic fluxes in the bacterium Escherichia coli. Nature 427:839-843

Almaas E, Oltvai ZN, Barabasi AL (2005) The activity reaction core and plasticity of metabolic networks. PLoS Comput Biol 1(7):e68

Barabasi AL, Oltvai ZN (2004) Network biology: understanding the cell's functional organization. Nat Rev Genet 5:101-113

Beard DA, Liang SD, Qian H (2002) Energy balance for analysis of complex metabolic networks. Biophys J 83(1):79-86

Burgard AP, Nikolaev EV, Schilling CH, Maranas CD (2004) Flux coupling analysis of genome-scale metabolic network reconstructions. Genome Res 14:301-312

Duarte NC, Herrgard MJ, Palsson BO (2004) Reconstruction and validation of Saccharomyces cerevisiae iND750, a fully compartmentalized genome-scale metabolic model. Genome Res 14:1298-1309
Edwards JS, Ibarra RU, Palsson BO (2001) In silico predictions of Escherichia coli metabolic capabilities are consistent with experimental data. Nat Biotechnol 19:125-130

Feist AM, Herrgard MJ, Thiele I, Reed JL, Palsson BO (2009) Reconstruction of biochemical networks in microorganisms. Nat Rev Microbiol 7(2):129-143

Fong SS, Joyce AR, Palsson BO (2005) Parallel adaptive evolution cultures of Escherichia coli lead to convergent growth phenotypes with different gene expression states. Genome Res 15(10):1365-1372

Hartwell LH, Hopfield JJ, Leibler S, Murray AW (1999) From molecular to modular cell biology. Nature 402:C47-C52

Ibarra RU, Edwards JS, Palsson BO (2002) Escherichia coli k-12 undergoes adaptive evolution to achieve in silico predicted optimal growth. Nature 420:186-189

Kauffman KJ, Prakash P, Edwards JS (2003) Advances in flux balance analysis. Curr Opin Biotechnol 14:491-496

Lee S, Phalakornkule C, Domach MM, Grossmann IE (2000) Recursive MILP model for finding all alternate optima in $1 p$ models for metabolic networks. Comput Chem Eng 24:711-716

Mahadevan R, Schilling CH (2003) The effects of alternate optimal solutions in constrained based genome-scale metabolic models. Metab Eng 5:264-276

Nishikawa T, Gulbahce N, Motter AE (2008) Spontaneous reaction silencing in metabolic optimization. PLoS Comput Biol 4(12): 1000236

Palumbo MC, Colosimo A, Giuliani A, Farina L (2005) Functional essentiality from topology features in metabolic networks: a case study in yeast. FEBS Lett 579:4642-4646

Price ND, Famili I, Beard DA, Palsson BO (2002) Extreme pathways and kirchhoff's second law. Biophys J 83(5):2879-2882

Price ND, Reed JL, Palsson BO (2004) Genome-scale models of microbial cells: evaluating the consequences of constraints. Nat Rev Microbiol 2:886-897

Reed JL, Palsson BO (2004) Genome-scale in silico models of E. coli have multiple equivalent phenotypic states: assessment of correlated reaction subsets that comprise network states. Genome Res 14:1797-1805

Reed JL, Vo TD, Schilling CH, Palsson BO (2003) An expanded genome-scale model of Escherichia coli k-12 (iJR904 GSM/ GPR). Genome Biol 4:R54

Samal A, Singh S, Giri V, Krishna S, Raghuram N, Jain S (2006) Low degree metabolites explain essential reactions and enhance modularity in biological networks. BMC Bioinform 7:118

Schuetz R, Kuepfer LUS (2007) Systematic evaluation of objective functions for predicting intracellular fluxes in Escherichia coli. Mol Syst Biol 3:119

Schuster S, Schuster R (1991) Detecting strictly detailed balanced subnetworks in open chemical reaction networks. J Math Chem 6:17-40

Schuster S, Pfeiffer T, Fell DA (2008) Is maximization of molar yield in metabolic networks favoured by evolution? J Theor Biol 252:497-504

Segre D, Vitkup D, Church GM (2002) Analysis of optimality in natural and perturbed metabolic networks. Proc Natl Acad Sci USA 99:15112-15117

Varma A, Palsson BO (1994) Metabolic flux balancing: basic concepts, scientific and practical use. Biotechnology 12:994-998

von Kamp A, Schuster S (2006) Metatool 5.0: fast and flexible elementary modes analysis. Bioinformatics 22(15):1930-1931

Wright J, Wagner A (2008) Exhaustive identification of steady state cycles in large stoichiometric networks. BMC Syst Biol 2:61 